\title{
Abnormal Median Normal Sural Sensory Response in a Patient with an Oropharyngeal Variant of Guillain-Barré Syndrome
}

\begin{abstract}
Yuu Yamazaki ${ }^{1}$, Tomohito Sugiura ${ }^{2}$ and Katsumi Kurokawa ${ }^{3}$
Key words: acute oropharyngeal palsy, acute inflammatory demyelinating polyneuropathy, anti-GQ1b antibody, anti-GT1a antibody, Campylobacter jejuni
\end{abstract}

(Inter Med 49: 519-520, 2010)

(DOI: 10.2169/internalmedicine.49.2939)
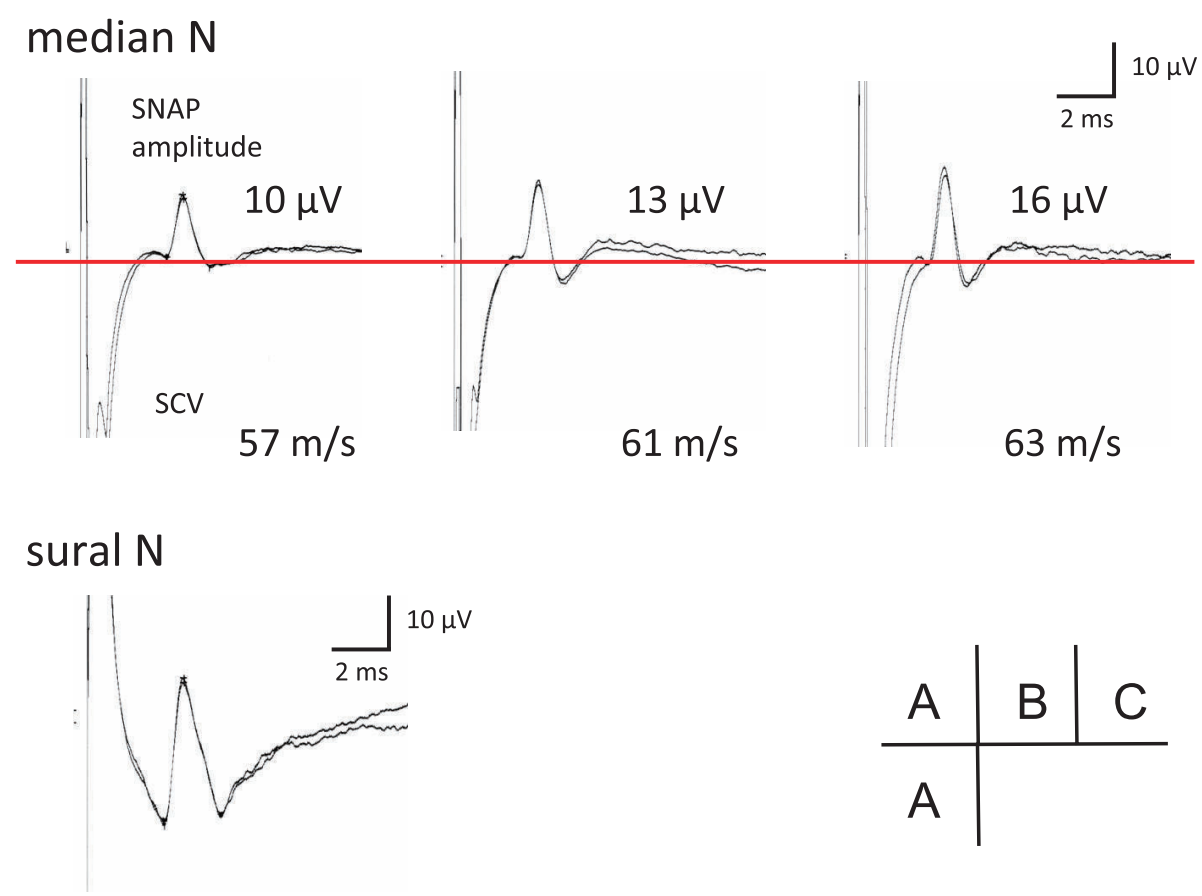

Picture 1. Sensory nerve conduction studies in the median $\mathbf{N}$ and sural N. Limb temperature was maintained above $32^{\circ} \mathrm{C}$ during the examination. The results on admission were as follows: median $\mathrm{N}$; SNAP amplitude $10 \mu \mathrm{V}$, SCV $56 \mathrm{~m} / \mathrm{s}$, sural N; SNAP amplitude $22 \mu \mathrm{V}$, SCV $46 \mathrm{~m} / \mathrm{s}$ (A). While distal SNAP amplitude of sural $N$ was within the normal range, that of median $N$ was beyond the laboratory normal limits (normal $>12 \mu \mathrm{V}$ ). Follow-up studies at one (B) and two (C) months later showed recovery and normalization of SNAP amplitude at the median $\mathrm{N}$. median $\mathrm{N}$ : median nerve, sural N: sural nerve, SNAP: sensory nerve action potential, SCV: sensory conduction velocity

\footnotetext{
${ }^{1}$ Department of Clinical Neuroscience and Therapeutics, Hiroshima University Graduate School of Biomedical Sciences, Hiroshima, ${ }^{2}$ Department of Neurology, National Hospital Organization Kure Medical Center and Chugoku Cancer Center, Kure and ${ }^{3}$ Division of Neurology, Department of Internal Medicine, Kawasaki Medical School, Kurashiki

Received for publication September 25, 2009; Accepted for publication November 24, 2009

Correspondence to Dr. Yuu Yamazaki, yuu.yamazaki@gmail.com
} 
were noted. Cerebrospinal fluid analysis revealed no pleocytosis but the protein content was $43 \mathrm{mg} / \mathrm{dL}$. Nerve conduction studies (NCS) were performed according to our standard methods. For the median sensory nerve conduction study, we used ring electrodes at the base of the index finger as stimulating electrodes. The exploring electrode was placed at the wrist $2.5 \mathrm{~cm}$ proximal to the wrist crease and the reference electrode was $3 \mathrm{~cm}$ proximal to the exploring electrode. For the sural nerve conduction study, the exploring electrode was placed at the optimal region between the ankle and heel, and the reference electrode was $3 \mathrm{~cm}$ distal to the exploring electrode. The sural nerve was stimulated at the calf portion $14 \mathrm{~cm}$ proximal to the exploring electrode. While motor nerve conductions were all within normal ranges, sensory nerve conductions showed 'abnormal median normal sural sensory response (AMNS)' pattern. Demyelinating neuropathy including lower cranial nerves was suspected. She received $40 \mathrm{mg} / \mathrm{kg} /$ day of gamma-globulin by intravenous administration (IVIG) for five consecutive days. Thereafter, her rhinolalia disappeared and she was discharged from hospital on the 7th day after admission. Soon after discharge, elevated serum anti-GQ1b and anti-GT1a IgG antibodies and isolation of Campylobacter jejuni from fecal culture confirmed the diagnosis of oropharyngeal variant of Guillain-Barré syndrome (GBS) $(1,2)$.

While GBS is divided into acute inflammatory demyelinating polyneuropathy (AIDP) or acute motor axonal neuropathy (AMAN) based on motor nerve conduction stud- ies (3), sensory nerve conduction studies also contribute to the electrodiagnosis of GBS in some cases (4). The AMNS pattern, reduced median amplitude compared with the sural amplitude, observed in the present patient is a key phenomenon highly suggestive of a primary demyelinating polyneuropathy (5).

Typical GBS is clinically characterized by the acute onset of generalized and symmetrical muscle weakness and areflexia from peripheral nerve involvement. In GBS-variants, however, some patients have unusual distribution of muscle involvement: O'Leary et al described three patients who had rapid progression of localized oropharyngeal weakness with or without ataxia (1). Unlike typical GBS or Fisher syndrome, these patients never developed limb weakness, ophthalmoplegia, or ptosis; they were regarded as having a regional variant of GBS based on the detection of anti-GQ1b and anti-GT1a IgG antibodies in serum. Another patient with acute oropharyngeal palsy was reported to have these antibodies with normal motor nerve conduction studies (2). In these variant cases, the involvement of one organ system can dominate the clinical picture so much that diagnostic difficulties of GBS can occur. Although the measurement of serum antiganglioside antibodies should prove useful for supporting the diagnosis of GBS or its variants (6), the assay may take several days to perform and it is costly. In the present case, the AMNS pattern supported the diagnosis and led us to perform earlier treatment for GBS.

\section{References}

1. O'Leary CP, Veitch J, Durward WF, Thomas AM, Rees JH, Willison HJ. Acute oropharyngeal palsy is associated with antibodies to GQ1b and GT1a gangliosides. J Neurol Neurosurg Psychiatry 61: 649-651, 1996.

2. Onodera M, Mori M, Koga M, et al. Acute isolated bulbar palsy with anti-GT1a IgG antibody subsequent to Campylobacter jejuni enteritis. J Neurol Sci 205: 83-84, 2002.

3. Kuwabara S. Guillain-Barré syndrome. Curr Neurol Neurosci Rep 7: 57-62, 2007
4. Kuwabara S, Ogawara K, Misawa S, et al. Sensory nerve conduction in demyelinating and axonal Guillain-Barré syndromes. Eur Neurol 51: 196-198, 2004.

5. Bromberg MB, Albers JW. Patterns of sensory nerve conduction abnormalities in demyelinating and axonal peripheral nerve disorders. Muscle Nerve 16: 262-266, 1993.

6. Koga M, Yuki N, Hirata K. Antiganglioside antibody in patients with Guillain-Barré syndrome who show bulbar palsy as an initial symptom. J Neurol Neurosurg Psychiatry 66: 513-516, 1999.

(C) 2010 The Japanese Society of Internal Medicine http://www.naika.or.jp/imindex.html 